\title{
Comparison of two non-primitive methods for path integral simulations: Higher-order corrections vs. an effective propagator approach
}

\author{
F. R. Krajewski and M. H. Müser \\ Inst. f. Physik, Johannes Gutenberg-Universität, 55099 Mainz, Germany
}

(October 30, 2018)

\begin{abstract}
Two methods are compared that are used in path integral simulations. Both methods aim to achieve faster convergence to the quantum limit than the socalled primitive algorithm (PA). One method, originally proposed by Takahashi and Imada, is based on a higher-order approximation (HOA) of the quantum mechanical density operator. The other method is based upon an effective propagator (EPr). This propagator is constructed such that it produces correctly one and two-particle imaginary time correlation functions in the limit of small densities even for finite Trotter numbers $P$. We discuss the conceptual differences between both methods and compare the convergence rate of both approaches. While the HOA method converges faster than the EPr approach, EPr gives surprisingly good estimates of thermal quantities already for $P=1$. Despite a significant improvement with respect to PA, neither HOA nor EPr overcomes the need to increase $P$ linearly with inverse temperature. We also derive the proper estimator for radial distribution functions for HOA based path integral simulations.
\end{abstract}

\section{INTRODUCTION}

Path integral Monte Carlo (PIMC) [1] and path integral molecular dynamics (PIMD) [2] have proven useful in the atomistic simulation of quantum effects occuring in condensed matter at low temperatures. The broad range of applications includes among other things superfluid ${ }^{4} \mathrm{He}$ [3], isotope effects in crystalline rare gas solids [4, 5] as well as phase transitions with strong quantum mechanical effects in Josephson junctions [6,7] and molecular solids [8,9]. Recently, path integral methods have also been applied to calculate low-temperature material properties of systems that are computationally less easily tractable than Lennard-Jones type systems: solid germanium [10], crystalline polyethylene [11], diamond [12], silica [13], and even Wigner crystals [14], to name a few.

A disadvantage of PIMC and PIMD is the increase of necessary computing time $t_{\mathrm{CPU}}$ with decreasing temperature $T$ at a given required accuracy. Using the so-called primitive approximation together with the most efficient sampling algorithms that completely eliminate critical slowing down (see Ref. [2] for a thorough discussion), it is not possible to overcome 
$t_{\mathrm{CPU}} \propto 1 / T$. Therefore different improvements on the primitive algorithms have been suggested that render path-integral simulations more efficient. One may subdivide the bulk of such attempts into three categories (i) methods that are based on higher-order approximants (HOA) of the high-temperature density matrix [15], (ii) methods that use effective propagators (EPr) that automatically yield the proper two-particle behavior [3].16], and (iii) methods that decompose the Hamiltonian into a harmonic and an anharmonic contribution before applying the Trotter formula [17, 18]. The latter category, also refered to as the effective potential (EP) method, will not be discussed any further in this study, mainly due to its unfavorable scaling of $t_{\mathrm{CPU}}$ with particle number $N$. Additionally, harmonic approximations are problematic for many systems of interest, in particular those involving ${ }^{3} \mathrm{He}$ and ${ }^{4} \mathrm{He}$. Yet, another advantage of HOA and EPr methods over the EP method is that the pathological behaviour of the attractive Coulomb potential is overcome automatically [16,19,20].

In this study, we want to compare the convergence of the HOA method, the EPr method, and the primitive algorithm for a simple model system. As long as the interaction potentials are well-behaved, the convergence does generally not depend on the specific form of the potential [21]. The test model system should of course be chosen such that it does not favor intrinsically one approach over the other, e.g., we may not chose a two-particle system, because then the EPr model would be exact per definition. As we are not interested in the EP approach, we can chose a simple monoatomic chain with harmonic next-neighbor coupling. This choice of system enables us to do the bulk of the calculations analytically so that statistical error bars are eliminated completely. We also want to investigate how the Trotter number $P$ necessary to keep the systematic errors below a well-defined percentage scales with inverse temperature for the different approaches investigated in this study.

It should be emphasized that the HOA method and the EPr method are conceptually different. In an EPr path integral simulation, one tries to generate radial distribution functions that are in the quantum limit (at least in a low-density approximation). Evaluating observables such as the thermal expectation value of the potential energy $\left\langle V_{\text {pot }}\right\rangle$ is done by simply evaluating the operator of the potential energy at the given distance. In a HOA path integral simulation, generalized estimators have to be defined even for those observables that are orthogonal in real space. We will comment on this in more depth in the following section. In particular we will derive an expression for the HOA estimator of the radial distribution function, which has not been given hitherto, which might explain the sparse use of the

method in the literature. The new estimator will be used to calculate the argon argon radial distribution function of those atoms in a simple three-dimensional Lennard-Jones crystal. The different methods employed in this study will be outlined in Sec. II. In this section we will also present a simplified approach to the EPr approach, which we call reduced effective propagator $(\mathrm{r}-\mathrm{EPr})$ approach. The results will be presented in Sec. III, and a short summary is given in Sec. IV.

\section{METHODS}

\section{A. Primitive Algorithm}

The primitive algorithm for path integrals is based on Feynman's idea to represent the partition function of a quantum mechanical point particle $Z(\beta)$ as a partition function of a 
classical ring polymer [22,23]. The potential $V_{\mathrm{rp}}(\{r\})$ of the classical ring polymer has the form:

$$
V_{\mathrm{rp}}(\{r\})=\sum_{t=1}^{P}\left[\frac{1}{2} \frac{m P^{2}}{\beta^{2} \hbar^{2}}\left(r_{t}-r_{t+1}\right)^{2}+V\left(r_{t}\right)\right]
$$

with $\beta=1 / k_{B} T$. $r_{t}$ represents the position of monomer $t$ in the ring polymer $\left(r_{t}=r_{t+P}\right)$, and $V$ is the real (physical) potential. $t$ is sometimes interpreted as an imaginary time and $P$ is commonly called the Trotter number. The PIMC or PIMD program are then assumed to generate distributions such that the probability of configuration $\{r\}$ to occur is proportional to $\exp \left[-\beta V_{\mathrm{rp}}(\{r\}) / P\right]$. All thermal expectation values of observables diagonal in real space can be determined directly from the configurations, e.g.,

$$
\langle V\rangle=\lim _{P \rightarrow \infty} \lim _{M \rightarrow \infty} \frac{1}{M P} \sum_{i=1}^{M} \sum_{t=1}^{P} V\left(r_{t, i}\right),
$$

where $r_{t, i}$ is the position of the $t^{\prime}$ th monomer in the $i$ 'th Monte Carlo step and $M$ is the number of observations in the Monte Carlo simulation. We refer to Refs. [1] for further details on the primitive algorithm.

\section{B. Higher-Order Approximant Method}

The HOA method is based on a fourth-order Hermitian Trotter decomposition of the high-temperature density matrix [24]. The decomposition was first applied to continuous degrees of freedom by Takahashi and Imada [15] as well as by Li and Broughton [19. The basic idea of the decomposition is to approximate the high-temperature density matrix $\hat{\rho}=\exp (-\beta \hat{H} / P)$ with

$$
\hat{\rho} \approx e^{-\beta \hat{V} / 2 P} e^{-\beta \hat{T} / 2 P} e^{-\beta \hat{V}_{\text {cor }} / P} e^{-\beta \hat{T} / 2 P} e^{-\beta \hat{V} / 2 P}
$$

where $\hat{H}=\hat{T}+\hat{V}$ corresponds to the Hamiltonian and $V_{\text {cor }}=\beta^{2}[[\hat{V}, \hat{T}], \hat{V}] / 24 P^{2}$ is a correction term. $\hat{T}$ and $\hat{V}$ are usually chosen to be kinetic and potential energy, respectively. For this decomposition, the correction energy $V_{\text {cor }}$ can be written as

$$
V_{\text {cor }}=\frac{\beta^{2} \hbar^{2}}{24 P^{2}} \sum_{n=1}^{N} \frac{1}{m_{n}}\left(\nabla_{n} V\right)^{2},
$$

where $m_{n}$ corresponds to the mass of particle $n$. Owing to the temperature-dependence of $V_{\text {cor }}$, thermal expectation values of observables $O$ have to be reevaluated with respect to the primitive algorithm, e.g., averages of functions diagonal in real space read

$$
\begin{aligned}
\langle\hat{O}\rangle= & \frac{1}{M P} \lim _{P \rightarrow \infty} \lim _{M \rightarrow \infty}\left[\sum_{i=1}^{M} \sum_{t=1}^{P}\{O(\{r\})\right. \\
& \left.\left.+\sum_{n=1}^{N} \frac{\beta^{2} \hbar^{2}}{12 m_{n} P^{2}}\left(\nabla_{n} V\right)\left(\nabla_{n} O\right)\right\}\right],
\end{aligned}
$$


where $V$ on the right-hand side of Eq. 5 only includes the original potential and not the correction term $V_{\text {cor }}$. The accuracy of the HOA method outlined above allows one to determine thermal expectation values with a leading correction of $1 / P^{4}$, while the primitive algorithm has leading corrections in the order of $1 / P^{2}$ [15, 19, 21.

Eq. (可) allows one easily to find the estimator for the potential energy to be $V+2 V_{\text {cor }}$, see Refs. [15, 19] for further details on the calculation of thermal expectation values. To our knowledge, however, it is has not yet been discussed that even the estimator for radial distribution functions $g(r)$ needs to be altered with respect to the primitive approach, for which the estimator can be written as

$$
g_{\text {prim }}^{\text {estim }}(r) \propto \delta\left(r-\left|\mathbf{r}_{t, i, n}-\mathbf{r}_{t, i, n^{\prime}}\right|\right) / r^{2},
$$

$\mathbf{r}_{t, i, n}$ denoting the position of particle $n$ of the $t$ 's monomer in ring polymer (particle) $n$. Applying Eq. (5) to Eq. (6) leads to a shift of the estimator for the distance between particle $n$ and $n^{\prime}$. Simply applying Eq. (5) to the operator for the square of the distance between particle $n$ and $n^{\prime}$ yields the estimator $r_{n, n^{\prime}}^{\text {estim }}$ for the distance between particle $n$ and $n^{\prime}$, which is found to be

$$
\begin{aligned}
r_{n, n^{\prime}}^{\mathrm{estim}} & =\left\{\left(\mathbf{r}_{t, i, n}-\mathbf{r}_{t, i, n^{\prime}}\right)^{2}\right. \\
& \left.+\frac{\beta^{2} \hbar^{2}}{6 P^{2}}\left(\frac{\nabla_{n} V}{m_{n}}-\frac{\nabla_{n^{\prime}} V}{m_{n^{\prime}}}\right)\left(\mathbf{r}_{t, i, n}-\mathbf{r}_{t, i, n^{\prime}}\right)\right\}^{1 / 2} .
\end{aligned}
$$

Thus $r_{n, n^{\prime}}^{\text {estim }}$ should replace $r$ in the argument of the $\delta$-function on the right hand side of Eq. (6) in order to calculate $g(r)$. Based on this relation, one may say that the HOA method is not an importance sampling algorithm in the sense that the probability for a configuration to occur in the simulation is proportional to the diagonal elements of $\hat{\rho}$ in a real space representation.

\section{Effective Propagator Approach}

An alternative non-primitive method to the HOA method is to construct effective potentials such that the two-particle propagators are reflected accurately, e.g., it is correct in all orders of $\hbar$. This effective potential is then used in a multi-particle simulation and hence produces the proper thermal behaviour in the low-density limit. No effective estimators have to be defined for observables diagonal in real space and in this sense, the approach is an importance sampling algorithm. The effective propagator (EPr) method is particularly useful for ill-behaved potentials such as the attractive Coulomb potential [16]. One may write the high-temperature two-particle density operator in the following way:

$$
\begin{aligned}
& \left\langle\mathbf{r}_{1} \mathbf{r}_{2}\left|e^{-\beta \hat{H} / P}\right| \mathbf{r}_{1}^{\prime} \mathbf{r}_{2}^{\prime}\right\rangle \propto \exp \left[-\frac{\beta}{P} V_{\text {eff }}\right] \\
& \times \exp \left[-\frac{\beta}{P} \frac{m P^{2}}{2 \beta^{2} \hbar^{2}}\left\{\left(\mathbf{r}_{1}-\mathbf{r}_{1}^{\prime}\right)^{2}+\left(\mathbf{r}_{2}-\mathbf{r}_{2}^{\prime}\right)^{2}\right\}\right] .
\end{aligned}
$$

$V_{\text {eff }}$ is a function that depends on $\mathbf{r}_{1}, \mathbf{r}_{1}^{\prime}, \mathbf{r}_{2}$, and $\mathbf{r}_{2}^{\prime}$. Therefore the interaction can be said to be non-local in imaginary time, e.g., in the primitive decomposition $\mathbf{r}_{1}$ does not couple 
directly to $\mathbf{r}_{2}^{\prime}$. The calculation of both the diagonal and the non-diagonal elements in more than one dimension is not trivial for non-harmonic potentials and we refer to Ref. [3] for an in-depth discussion of that problem. For our one-dimensional model system, however, the approach can be simplified significantly, i.e., it can be solved analytically up to a summation over finite number of terms. This will be done in the Section III].

\section{The r-EPr method}

In general the implementation of the full two-particle pair propagator with correct diagonal and non-diagonal elements is difficult because, one has to use the two-particle hightemperature density matrix (HTDM) $\left\langle\mathbf{r}_{1} \mathbf{r}_{2}\left|e^{-\beta \hat{H} / P}\right| \mathbf{r}_{1}^{\prime} \mathbf{r}_{2}^{\prime}\right\rangle$. In principle one can evaluate this expression prior to the simulation. However, this is a difficult task due to the dimensionality of the matrix, in particular for anharmonic $d=3$ dimensional systems. Even if one expresses the HTDM in the center of mass system of the coordinates $\mathbf{r}_{1}, \mathbf{r}_{2}, \mathbf{r}_{1}^{\prime}, \mathbf{r}_{2}^{\prime}$ and even if one uses an appropriate orientation of the axes within a molecular-fixed frame, one is a left with a three dimensional table and transformations between molecular fixed frames and laboratory system. It is also difficult to find good fit functions for the effective potential $V_{\text {eff. }}$

The main idea of a reduced effective potential approach (r-EPr) is to only incorporate those corrections to the primitive decomposition that are local, e.g., those corrections that involve coordinates at different Trotter indices are neglected. In other words, if one carries out a simulation at inverse temperature $\beta$ with Trotter number $P$, one uses an effectively classical potential that reproduces the correct two-particle distribution function in the lowdensity limit at inverse temperature $\beta / P$. This approach is similar to an idea suggested by Thirumalai et al. 25], who constructed effective interaction potentials from the diagonal elements of the high-temperature density matrix, see also a related paper by Pollock and Ceperley [26].

\section{RESULTS}

\section{A. Linear, harmonic chain}

In order to analyze the convergence of primitive algorithm, HOA and EPr method respectively, we chose a one-dimensional linear chain with harmonic next neighbor coupling

$$
V=\frac{1}{2} \sum_{n=1}^{N} k\left(r_{n}-r_{n+1}\right)^{2} .
$$

Periodic boundary conditions, $r_{N+1}=r_{1}$ are applied and the masses $m$ are identical for all atoms.

HOA and EPr invoke correction terms in the potential energy of the ring polymers with respect to the original expression of the primitive approach that is given in Eq. (1) for a one particle problem. All three approaches can be represented as the limiting case of a $(1+1)$ dimensional solid with harmonic coupling between nearest neighbors, next nearest and next next nearest neighbors. A graphical illustration is given in Figure 1. 
The new effective energy $\tilde{V}_{\text {rp }}$ that enters the Boltzmann factor reads:

$$
\begin{aligned}
\tilde{V}_{\mathrm{rp}}= & \frac{1}{2} \sum_{n=1}^{N} \sum_{t=1}^{P} \sum_{ \pm}\left[\left(\kappa+\tilde{\kappa}_{1}\right)\left(r_{t, n}-r_{t+1, n}\right)^{2}\right. \\
& +\left(k+\tilde{k}_{1}\right)\left(r_{t, n}-r_{t, n+1}\right)^{2}+\tilde{\kappa}_{2}\left(r_{t, n}-r_{t \pm 1, n+1}\right)^{2} \\
& \left.+\tilde{k}_{2}\left(r_{t, n}-r_{t, n+2}\right)^{2}\right] .
\end{aligned}
$$

The expression $\tilde{V}_{\text {rp }}$ can be diagonalized in terms of Fourier modes

$$
\tilde{r}_{q, \omega}=\sqrt{\frac{1}{P N}} \sum_{t=1}^{P} \sum_{n=1}^{N} r_{t, n} e^{2 \pi i q n / N} e^{2 \pi i \omega t / P} .
$$

It is convenient to introduce $k^{\prime}=k+\tilde{k}_{1}$ and $\kappa^{\prime}=\kappa+\tilde{\kappa}_{1}$ with $\kappa=m P^{2} /\left(\beta^{2} \hbar^{2}\right)$ in order to reexpress $\tilde{V}_{\text {rp }}$ as

$$
\tilde{V}_{\mathrm{rp}}=\frac{1}{2} \sum_{\omega=1}^{P} \sum_{q=1}^{N} k_{q, \omega}\left|\tilde{r}_{q, \omega}\right|^{2}
$$

with

$$
\begin{aligned}
\tilde{k}_{q, \omega}= & \kappa^{\prime} 4 \sin ^{2}\left(\frac{\pi}{P} \omega\right)+k^{\prime} 4 \sin ^{2}\left(\frac{\pi}{N} q\right) \\
& +\tilde{\kappa}_{2} 4\left[\operatorname { s i n } ^ { 2 } \left(\pi(q / N+\omega / P)+\sin ^{2}(\pi(q / N-\omega / P)]\right.\right. \\
& +\tilde{k}_{2} 4 \sin ^{2}\left(\frac{\pi}{N} 2 q\right) .
\end{aligned}
$$

The partition function $Z_{c}$ for the classical system illustrated in Fig. 1 1 can then be reduced to NP Gaussian integrals. $Z_{c}$ is proportional to

$$
Z_{c} \propto \prod_{q=1}^{N} \prod_{\omega=1}^{P}{\sqrt{\tilde{k}_{q, \omega}}}^{-1} .
$$

For the different algorithms we find different functions for $\tilde{k}_{1}, \tilde{k}_{2}, \tilde{\kappa}_{1}$, and $\tilde{\kappa}_{2}$. The expressions

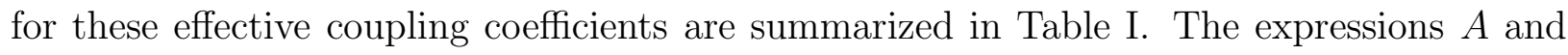
$C$ used in Table [ for the EPr treatment will be given and derived below.

We will now calculate the thermal expectation value of the potential energy $\left\langle V_{P}\right\rangle$ for a given Trotter number $P$. One can expect that the convergence rate does not depend on the observable, which is why it is sufficient to only investigate $\left\langle V_{P}\right\rangle$. The calculations for the three approaches will be separated into three subchapters.

\section{Solution for the primitive Method}

In the primitive method, the usual thermodynamic relationships can be used in a straightforward way without modification, albeit their use can be impractical for technical reasons. In order to calculate the thermal expectation value of $\left\langle V_{P}\right\rangle$, one can use the relation: 


$$
\left\langle V_{P}\right\rangle=-\frac{k}{\beta} \frac{d}{d k} \ln \left(Z_{c}\right),
$$

where all effective coupling coefficients vanish. This relationship simply follows from the formal expression for the quantum mechanical partition function of a linear, monoatomic harmonic chain. The final expression for $\left\langle V_{P}\right\rangle$ then is:

$$
\left\langle V_{P}\right\rangle=\frac{k}{2 \beta} \sum_{q=1}^{N} \sum_{\omega=1}^{P} \frac{4 \sin ^{2}(\pi q / N)}{\tilde{k}_{q, \omega}} .
$$

This equation can be interpreted as follows: Equipartition requires that $\tilde{k}_{q, \omega}\left|x_{q, \omega}\right|^{2}=k_{B} T$. Hence $\tilde{k}_{q, \omega}$ appears in the denominator. The amount of real potential energy in these modes, however, is only $\tilde{k}_{q}\left|x_{q, \omega}\right|^{2} / 2$, where $\tilde{k}_{q}$ is the stiffnes that can be associated with the mode $q$ in a classical linear chain.

\section{Solution for the HOA method}

Eq. (15) also holds for the HOA method, because the only modification with respect to $\mathrm{PA}$ is that a better approximant for the high-temperature density matrix is emploied. However, the correct coupling coefficients $\tilde{k}_{1}$ and $\tilde{k}_{2}$ have to be used. The expressions for

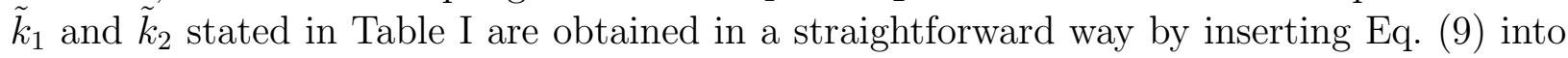
Eq. (四). This leads to the expression

$$
\begin{aligned}
\left\langle V_{P}\right\rangle= & \frac{k}{2 \beta} \sum_{q=1}^{N} \sum_{\omega=1}^{P}\left\{\left(1+\frac{2}{3} \frac{\hbar^{2}}{m}\left(\frac{\beta}{P}\right)^{2} k\right) 4 \sin ^{2}\left(\frac{\pi}{N} q\right)\right. \\
& \left.-\frac{2}{3} \frac{\hbar^{2}}{m}\left(\frac{\beta}{P}\right)^{2} k \sin ^{2}\left(\frac{\pi}{N} 2 q\right)\right\} / \tilde{k}_{q, \omega}^{\mathrm{HOA}},
\end{aligned}
$$

where $\tilde{k}_{q, \omega}^{\mathrm{HOA}}$ refers to that expression for $\tilde{k}_{q, \omega}$ in Eq. (13) which is obtained by inserting the HOA values for $\tilde{k}_{1}$ and $\tilde{k}_{2}$. The same result for $\left\langle V_{P}\right\rangle$ could have been obtained by calculating the second moments of the eigenmodes $\left\langle\left|\tilde{x}_{q, \omega}\right|^{2}\right\rangle$ from equipartition. The resulting $\left\langle\left|\tilde{x}_{q, \omega}\right|^{2}\right\rangle$ could then have been used to calculate the proper HOA potential energy estimator $V+2 V_{\text {cor }}$.

\section{Solution for the EPr method}

In the EPr approach, $Z_{c}$ does not follow from a Hermitian decomposition of the hightemperature density matrix. Therefore Eq. (15) can not be emploied for the calculation of $\left\langle V_{P}\right\rangle$. However, we can use the fact that EPr is an importance sampling method. Since we can obtain $\left\langle\left|\tilde{x}_{q, \omega}\right|^{2}\right\rangle$ from equipartition and since the real potential energy of mode $q$ in a (classical) linear chain is proportional to $k \sin ^{2}(\pi q / N)$ ), it is possible to use Eq. (16) where the denominator $\tilde{k}_{q, \omega}$ is taken from the EPr column in Table $\llbracket$. Hence,

$$
\left\langle V_{P}\right\rangle=\frac{k}{2 \beta} \sum_{q=1}^{N} \sum_{\omega=1}^{P} \frac{4 \sin ^{2}(\pi q / N)}{\tilde{k}_{q, \omega}^{\mathrm{EPr}}} .
$$


We are now concerned with the derivation of the expressions for $A$ and $C$ in Table $\mathbb{E}$. To do this, one has to consider a dimer in which the two atoms are coupled by a harmonic spring of stiffness $k$. One then transforms the dimer Hamiltonian into the center-of-mass system with $R=\left(r_{1}+r_{2}\right) / 2$ the center-of-mass coordinate and $\Delta R=\left(r_{1}-r_{2}\right)$ the reduced distance between the two atoms. These two coordinates can be associated with two modes, the center of mass mode with mass $M=2 m$ and the internal oscillator mode with stiffnes $k$ and reduced mass $\mu=m / 2$. Both the free particle density matrix and the high-temperatur density matrix (HTDM) of an oscillator with spring constant $k$ and mass $\mu$ are known exactly. The free particle HTDM is simply proportional to $\exp \left[-M P\left(R-R^{\prime}\right)^{2} / 2 \beta \hbar^{2}\right]$ (see Eq. (11)) while the oscillator's HTDM is given by 223:

$$
\begin{aligned}
& \rho\left(\Delta R, \Delta R^{\prime}, \beta / P\right)=\sqrt{\frac{\sqrt{\mu k}}{2 \pi \hbar \sinh (2 f)}} \times \\
& \exp \left\{\frac{-\sqrt{\mu k}}{2 \hbar \sinh (f)}\left[\left(\Delta R^{2}+\Delta R^{\prime 2}\right) \cosh (f)-2 \Delta R \Delta R^{\prime}\right]\right\} .
\end{aligned}
$$

The prefactor on the right-hand side of Eq. (19) provides an irrelevant offset in $V_{\text {eff }}$, which will be neglected in the following treatment. One then needs to transform the product of the free HTDM and the internal oscillator HTDM back into the laboratory system and express the effective potential according to Eq. (8). With the definition of

$$
f=\frac{\beta}{P} \hbar \sqrt{\frac{2 k}{m}}
$$

we obtain the parameters $A$ and $C$

$$
\begin{aligned}
& A=\frac{2}{f} \tanh \left(\frac{f}{2}\right), \\
& C=\frac{2}{f^{2}}\left\{1-\frac{f}{\sinh (f)}\right\}
\end{aligned}
$$

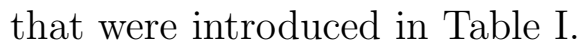

\section{Solution for the r-EPr approach}

According to Sec. [IC 1, we need to know the quantum-mechanical radial distribution function $g(r)$ at inverse temperature $\beta / P$ for the reduced harmonic oscillator in order to construct the r-EPr effective potential $V_{\text {eff }}$. For this purpose, it is sufficient to know the internal energy of the reduced harmonic oscillator, since $g(r)$ is a simple Gaussian at all temperatures. Hence we need to find the effective coupling constant $k^{\prime}$ which generates a second moment $\left\langle x^{2}\right\rangle$ in a classical treatment at temperature PT such that the the real potential energy $k\left\langle x^{2}\right\rangle / 2$ corresponds to the quantum limit at temperature $P T$. This condition, which defines $k^{\prime}$, can be written as

$$
\langle V(\beta / P)\rangle_{\text {exact }}=\frac{1}{2} \frac{k}{k^{\prime}} k_{B} T P,
$$


where $\langle V(\beta / P)\rangle_{\text {exact }}$ is the correct thermal potential energy for an oscillator at temperature $P T$. For a harmonmic oscillator, $\langle V(\beta / P)\rangle_{\text {exact }}$ is half the internal energy $U$, given by $U=0.5 \hbar \omega \operatorname{coth}(\beta \hbar \omega / 2)$ with frequency $\omega=\sqrt{k / \mu}=\sqrt{2 k / m}$. We solve for $k^{\prime}$ and find $k^{\prime}=A k$. The parameter $A$ is given in Eq. (21) and $\tilde{k}_{1}=k(A-1)$ as stated in Table $\mathbb{\|}$ follows.

\section{Comparison of the methods}

The main issue of this study is the analysis of the convergence of thermal expectation values such as the potential energy $\left\langle V_{P}\right\rangle$ to the proper quantum limit as a function of Trotter number $P$. We consider a linear chain consisting of $N=5$ atoms and periodic boundary conditions. The convergence does not depend on $N$ in a qualitative way. It is examined at a fixed thermal energy well below the Debye frequency of the chain, namely at inverse temperature $\beta=64 /(\hbar \sqrt{k / m})$. A linear plot of $\left\langle V_{P}\right\rangle$ is shown in Fig. 22.

It can be seen that at $P=1$ the EPr and the r-EPr method start off with estimates that are very close to the quantum limit while PA and HOA start off with an estimate near the classical value. Upon increasing $P$ the EPr approaches the proper value from below, while for the r-EPr method the deviation between estimate and proper result first increases before it decreases again. At a Trotter number $P \approx 64$, the HOA method becomes similarly good as the EPr approach. In order to address the convergence in a more quantitative way, it is convenient to analyze the relative deviation of $\left\langle V_{P}\right\rangle$ from the exact value $\left\langle V_{E}\right\rangle$ as a function of $P$ in a double logarithmic plot, which is done in Fig. 3 .

It can be seen that at large Trotter numbers the HOA method converges with $P^{-4}$ to the quantum limit, while all other methods only converge with $P^{-2}$. The prefactor for the EPr, however, is much smaller than for r-EPr and PA. The value of $P$ at which convergence starts is similar in all approaches, e.g., $k_{B} T P$ is larger than but in the order of $\hbar \sqrt{k / m}$. The accuracy where HOA becomes better than EPr is 1.7\%. For this particular model system, this value of $1.7 \%$ was found to be independent of temperature. We expect it to be similar for all systems that are dominated by harmonic interactions. We will now address the question how we have to increase $P$ for the various approaches if we lower $T$ and require the relative accuracy to be constant, e.g., 1\%. The results are shown in Fig. 4 .

In order for the relative error to be constant, all methods require that $P$ increases linearly with inverse temperature $\beta$. The r-EPr approach, which is a little more difficult to implement than the PA method, requires slightly reduced Trotter numbers with respect to $P A$. We want to emphasize that the behavior shown in Fig. 4 is qualitatively similar if the accuracy criterion for $P$ is changed, however, the stricter the criterion the larger the gap between EPr and HOA. This trend can be seen in Fig. 四), where we require one time $0.1 \%$ accuracy instead of $1 \%$ as shown in Fig. (4a). Only if one is confined to the use of very small $P$, EPr might be the better choice. One may conclude that the optimal method depends on the desired accuracy. 


\section{B. Crystalline Argon}

It has been pointed out in Section $1 \mathrm{~B}$ that in order to calculate radial correlation functions, it is necessary to correct the estimator for $g(r)$. An important question to address is how well the HOA approach allows one to calculate $g(r)$. In order to examine this issue we apply the HOA method (making use of the proper estimator, see Eq. (])) to crystalline argon. The results are presented in Fig. 5.

It is interesting to note that $g(r)$ is too broadened for the HOA approach while it is too narrow using the PA algorithm. Obviously, the agreement of the $P=12 \mathrm{HOA}$ calculation is already very close to the quantum limit. This is a little surprising as the product of $k_{B} T P$ is still far below the thermal energy of the Debye temperature, which is about $T_{D} \approx 70 \mathrm{~K}$. We want to note that if the $g(r)$ are obtained without corrections, the agreement is distinctly reduced. This might be the reason why the convergence of $g(r)$ reported by Li and Broughton for the attractive Coulomb potential was so slow [19]. In our case, omitting the corrections to the $g(r)$ estimator leads to peaks in $g(r)$ that are even sharper than those obtained with PA using identical Trotter numbers.

\section{SUMMARY}

In this study we have compared the convergence to the quantum limit for different path integral approaches. As the convergence rate does not depend on the specific model system (as long as the potentials are well-behaved), we have focused our attention to a linear chain of harmonically coupled atoms. We disregarded methods making explicit use of a decomposition into a harmonic and an anharmonic part of the Hamiltonian among other reasons due to the unfavorable scaling of the numerical effort with system size.

The three main approaches investigated were the so-called primitive algorithm (PA), a method based on a higher-order approximant (HOA), and an approach in which an effective potential is constructed such that the one and two-particle high-temperature density matrices are reproduced exactly in the limit of small densities. We called this approach the effective propagator (EPr) method. The latter approach can be further reduced by only taking into account corrections that are local in imaginary time leading to the r-EPr method.

We emphasized the different spirit of EPr and HOA methods: In the EPr method, observables orthogonal in real space can be evaluated directly, whereas the HOA method requires effective estimators. In this sense, HOA can be seen as a non-importance sampling technique that requires the use of effective estimators even for radial distribution functions. The need to alter estimators for radial distribution functions obtained in simulations taking into account quantum effects in an effective potential had not yet been discussed hitherto. We showed that the proper estimators lead to a very fast convergence to the quantum limit in a simple argon crystal.

For the linear monoatomic chain investigated in this study, we found that the corrections based on HOA vanish with $P^{-4}$ while all other methods, PA, EPr, and r-EPr, have corrections in the order of $P^{-2}$. The prefactor is similar for PA and r-EPr and distinctly smaller for EPr. Both EPr and r-EPr, however, give rather accurate results at small $P$, e.g., the relative error in the thermal expectation value of the potential energy is smaller than $10 \%$ in those approaches, while PA and HOA differ by nearly $100 \%$ at low temperatures and 
$P=1$. The Trotter number where convergence starts is similar in all approaches. If one requires high accuracy, e.g. $1 \%$, one needs to increase the Trotter number $P$ linearly in all approaches.

What can we conclude for path integral simulations? The use of HOA is certainly a little more (about twice) CPU time expensive than that of EPr, r-EPr, and PA (which all require approximataly the same amount of computing). This is because in an HOA based simulation, we need derivatives of the interaction potential that are one order higher than those in simulations based on EPr, r-EPr, and PA. However, one is rewarded with the best convergence to the quantum limit in a HOA based path integral simulation. While EPr also results in a significant improvement with respect to $\mathrm{PA}$, it is plagued with a tedious, non-trivial implementation procedure. Thus HOA should be the method of choice unless many-body correlations do not play a significant role like in gaseous helium.

\section{ACKNOWLEDGMENTS}

We thank Kurt Binder for useful discussions. Support from the BMBF through Grant 03N6015 and from the Materialwissenschaftliche Forschungszentrum Rheinland-Pfalz is gratefully acknowledged. 


\section{REFERENCES}

[1] J. A. Barker, J. Chem. Phys. 70, 2914 (1979).

[2] M. E. Tuckerman, B. J. Berne, G. J. Martyna, and M. L. Klein, J. Chem. Phys. 99, 2796 (1993).

[3] D. M. Ceperley, Rev. Mod. Phys. 67, 279 (1995).

[4] J.-L. Barrat, P. Loubeyre, and M. L. Klein, J. Chem. Phys. 90, 5644 (1989).

[5] M. H. Müser, P. Nielaba, and K. Binder, Phys. Rev. B 51, 2723 (1995).

[6] L. Jacobs, J. V. José, M. A. Novotny, and A. M. Goldman, Europhys. Lett. 3, 1295 (1987).

[7] L. Jacobs, J. V. José, M. A. Novotny, and A. M. Goldman, Phys. Rev. B 38, 4562 (1988).

[8] K. J. Runge, M. P. Surh, C. Mailhiot, and E. L. Pollock, Phys. Rev. Lett. 69, 3527 (1992).

[9] M. H. Müser and B. J. Berne, Phys. Rev. Lett. 77, 2638 (1996).

[10] J. C. Noya, C. P. Herrero, and R. Ramirez, Phys. Rev. B 56237 (1997).

[11] R. Martonak, W. Paul, and K. Binder, Phys. Rev. E 572425 (1998).

[12] C. P. Herrero and R. Ramirez, Phys Rev B 63, 024103 (2001).

[13] M. H. Müser, J. Chem. Phys. 114, 6364 (2001).

[14] L. Candido, P. Phillips, D. M. Ceperley, Phys. Rev. Lett. 86, 492 (2001).

[15] M. Takahashi and M. Imada, J. Phys. Soc. Jpn. 53, 3765 (1984).

[16] E. L. Pollock, Comput. Phys. Commun. 52, 49 (1988).

[17] R. Giachetti and V. Tognetti, Phys. Rev. B 33, 7647 (1986).

[18] A. Cuccoli, A. Macchi, V. Tognetti, and R. Vaia, Phys. Rev. B 47, 14923 (1993).

[19] X.-P. Li and J. Q. Broughton, J. Chem. Phys. 86, 5094 (1987).

[20] M. H. Müser and B. J. Berne, J. Chem. Phys. 107, 571 (1997).

[21] M. Suzuki, in Quantum Monte Carlo methods, Springer Series in Solid-State Sciences 74 (Springer, Berlin, 1987).

[22] R. P. Feynman and A. R. Hibbs, Quantum Mechanics and Path Integrals, (Mc.GrawHill, New York, 1965).

[23] R. P. Feynman, Statistical Mechanics, (W. A. Benjamin, Inc., 1972).

[24] H. De Raedt and B. De Raedt, Phys. Rev. A 28, 3575 (1983).

[25] D. Thirumalai, R. W. Hall, and B. J. Berne, J. Chem. Phys. 81, 2523 (1984).

[26] E. L. Pollock and D. M. Ceperley, Phys. Rev. B 30, 2555 (1984). 


\section{TABLES}

\begin{tabular}{c|cccc}
\hline \hline & PA & HOA & EPr & r-EPr \\
\hline$\tilde{k}_{1}$ & 0 & $(\hbar \beta k)^{2} /\left(3 m P^{2}\right)$ & $k(A-C-1)$ & $k(A-1)$ \\
$\tilde{k}_{2}$ & 0 & $(\hbar \beta k)^{2} /\left(12 m P^{2}\right)$ & 0 & 0 \\
$\tilde{\kappa}_{1}$ & 0 & 0 & $-k C$ & 0 \\
$\tilde{\kappa}_{2}$ & 0 & 0 & $\frac{1}{2} k C$ & 0 \\
\hline \hline
\end{tabular}

TABLE I. Expressions for the effective coupling coefficients that are represented in Fig. 1 


\section{FIGURES}

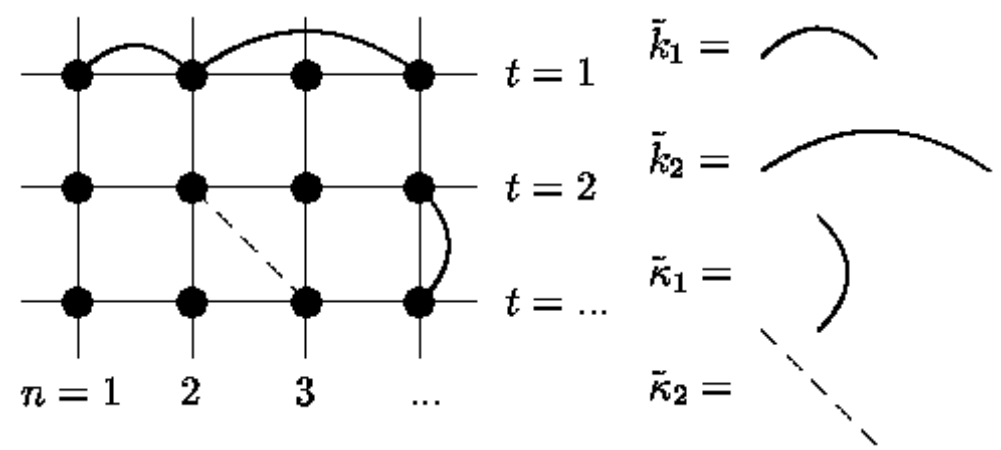

FIG. 1. Illustration of coupling between atoms. Atoms can only oscillate in horizontal direction. Vertical springs also act in horizontal direction. The straight horizontal lines represent springs between nearest neighbors of stiffness $k$, the solid vertical lines springs of stiffness $m k_{b}^{2} T^{2} P^{2} / \hbar^{2}$. 


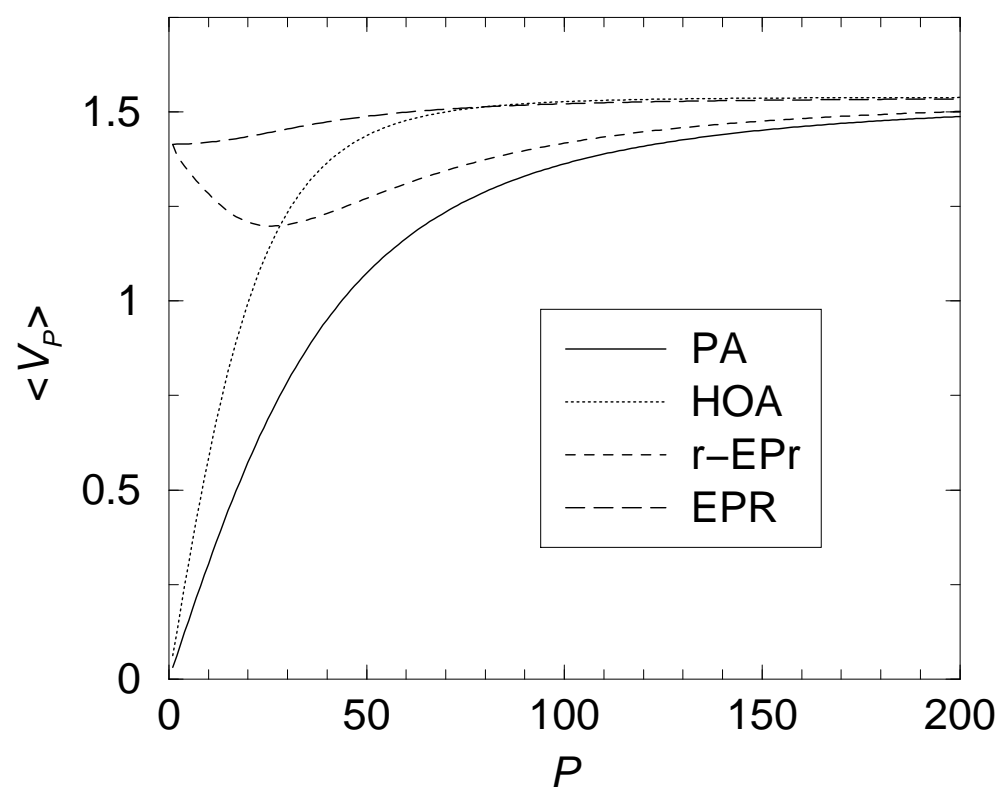

FIG. 2. Thermal expectation value of the potential energy $\left\langle V_{P}\right\rangle$ as a function of Trotter number $P ; \beta \hbar \sqrt{k / m}=64$.

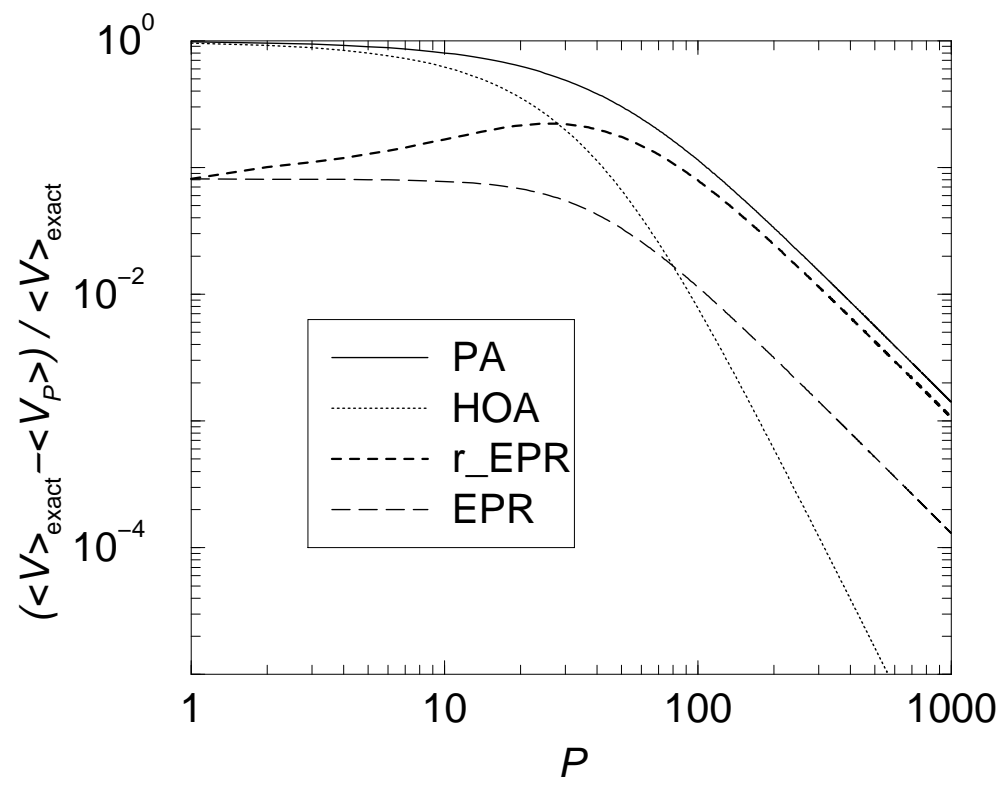

FIG. 3. Relative error of the potential energy for an $N=5$ chain at $\beta \hbar \omega=64$ as a function of Trotter number $P$. 

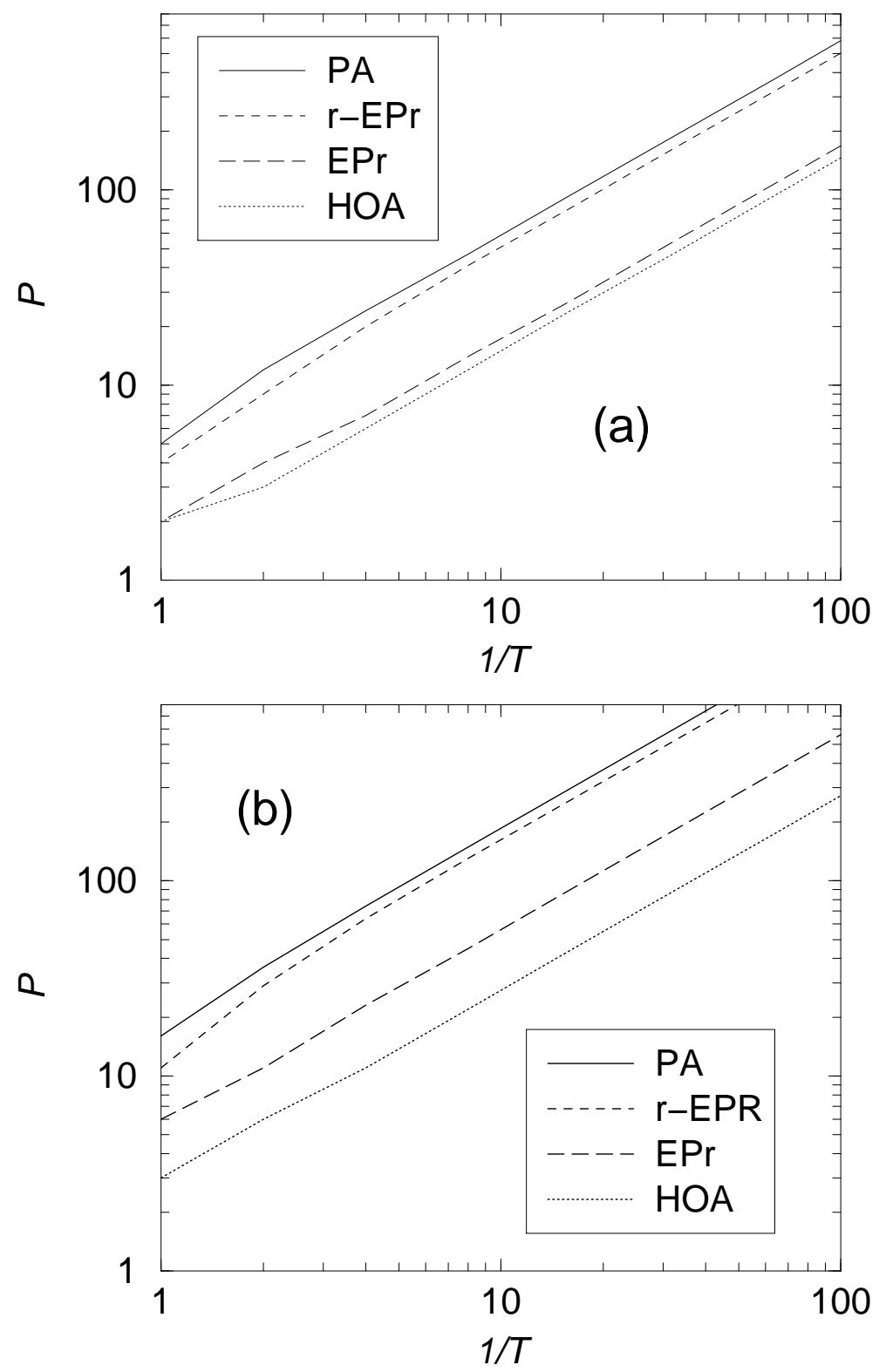

FIG. 4. Necessary Trotter number $P$ to reach a relative accuracy of a) $10^{-2}$ and b) $10^{-3}$ in the potential energy $\left\langle V_{P}\right\rangle$ at different inverse temperatures $1 / T$ for the linear chain consisting of $N=5$ atoms. 


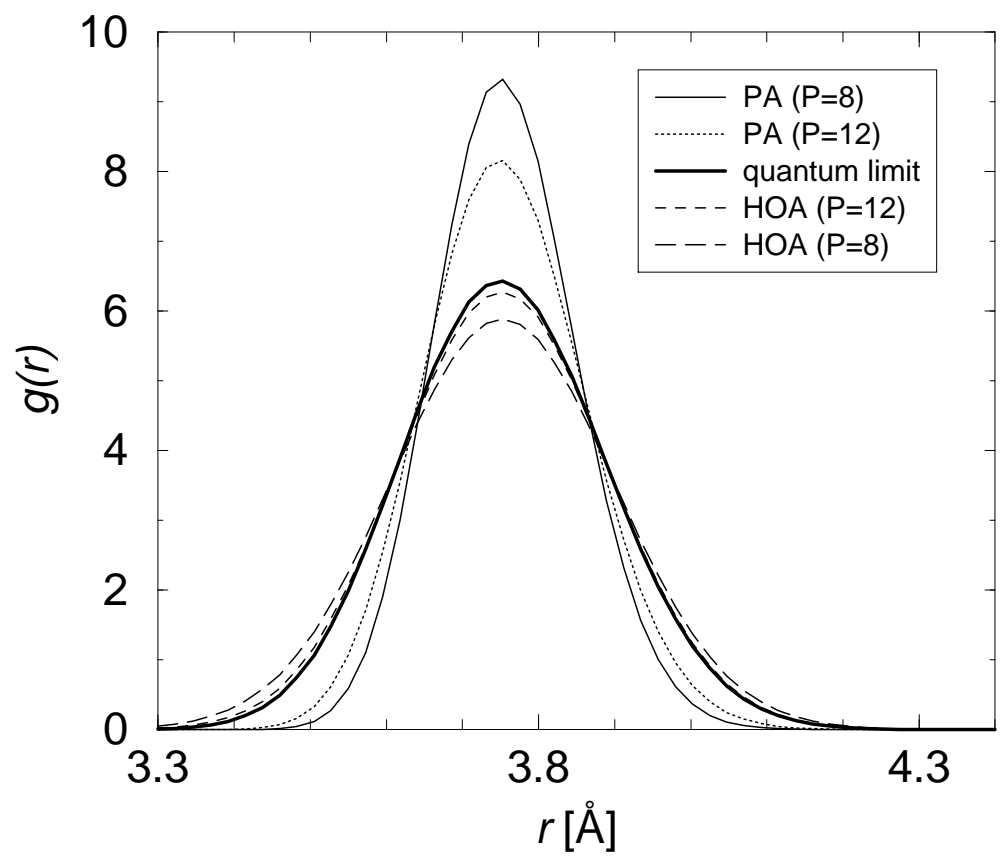

FIG. 5. Pair correlation function $g(r)$ of crystalline argon at $T=2 \mathrm{~K}$ calculated with the PA and HOA algorithm for Trotter numbers $P=8$ and $P=12$. As a reference a quasi-exact correlation function (HOA, $P=256)$ is included. 